\title{
W-Band GaAs HEMT MMIC Subharmonically Pumped Diode Mixers with 20 GHz IF Bandwidth
}

\author{
Yuh-Jing Hwang $^{(1)(2)}$, Huei Wang ${ }^{(1)}$, and Tah-Hsiung $\mathrm{Chu}^{(1)(2)}$
}

${ }^{(1)}$ Department of Communication Engineering and Department of Electrical Engineering, National Taiwan University, 1 Roosevelt Rd., Sec. 4, Taipei, 106, Taiwan, R.O.C.

(2) Academia Sinica Institute of Astronomy \& Astrophysics, P.O. Box 23-141, Taipei, 106, Taiwan, R.O.C.

Email: yjhwang@asiaa.sinica.edu.tw, hueiwang@ew.ee.ntu.edu.tw, thc@ew.ee.ntu.edu.tw

Two subharmonically pumped (SHP) diode mixers are designed for wideband W-band RF frequencies,

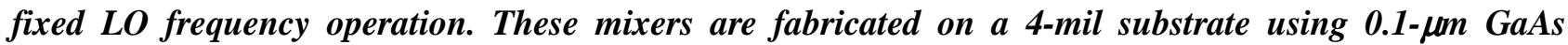
MMIC process. Both simulation and test results show that the mixers are with 12.25 and 11.75 dB average conversion losses, respectively. Both mixers have IF bandwidth wider than $20 \mathrm{GHz}$. The conversion loss flatness of the symmetric circuit is within $\pm 1.25 \mathrm{~dB}$. To our knowledge, these are the state-of-the-art result on low-conversion-loss wideband MMIC SHP diode mixers.

\section{INTRODUCTION}

Radio astronomy receivers require wide bandwidth to search the spectrum of molecular species in space with different redshift [1], or to observe the wideband continuum radiation, e.g. cosmic background radiation. In the past the high-performance heterodyne receivers are based on superconductor-insulator-superconductor mixers with only several $\mathrm{GHz}$ IF bandwidth. Recent progress on the development of cryogenic millimeterwave HEMT low-noise amplifiers makes the LNAbased wideband heterodyne receivers for MMW astronomy possible [2]-[5]. Wideband mixers are essential components for those LNA-based heterodyne receivers. For the MMW astronomical receiver application, we design SHP diode mixers with $20-\mathrm{GHz}$ IF bandwidth and only $\pm 1.25 \mathrm{~dB}$ conversion loss flatness at RF frequency range between $85-105 \mathrm{GHz}$ with fixed LO frequency at $42 \mathrm{GHz}$.

\section{CIRCUIT DESIGN}

The W-band SHP mixers utilized anti-parallel HEMT diode pairs are designed to down-convert 85 to $105 \mathrm{GHz}$ RF signal to IF frequency of 1 to $21 \mathrm{GHz}$ by mixing with the second harmonic of a 42-GHz LO signal. The mixer designs are fabricated by a GaAs PHEMT MMIC process on 4-mil substrate, using diodes with $0.1-\mu \mathrm{m}$ gates length and $16-\mu \mathrm{m}$ total periphery.

In order to achieve the widest IF frequency bandwidth on the upper sideband, we explore two slightly different circuit structures. The first design, with chip named WSHM1, is adapted from the typical SHP mixer circuit structure [6][7], with simple RF-IF diplexer circuit consists of a coupled-line RF band-pass filter and a step impedance line section IF low-pass filter. Near the diode pair, a LO frequency quarter-wavelength open stub at RF input and a RF half-wavelength short-circuit stub at LO input are used to improve the isolation between RF and LO.

The other mixer design, WSHM2, is adapted from another circuit structures [8]-[10]. This design emphasizes the RF and LO circuit symmetry near the diode pair. In order to get better RF-IF isolation, we arrange two RF quarter wavelength sections along the IF matching network, with one in series and the other shunt in half way. Over the desired RF frequency band, this arrangement keeps the input impedance at the shunt point almost short circuit, while the IF input impedance at the RF-IF connecting junction looks almost open circuit, which establishes good RF-IF isolation inherently. Stepped- impedance IF low-pass filter consists of the in-series RF quarter wavelength section to reduce the size. Both MMIC chip layouts are shown on Figure 1 and Figure 2.

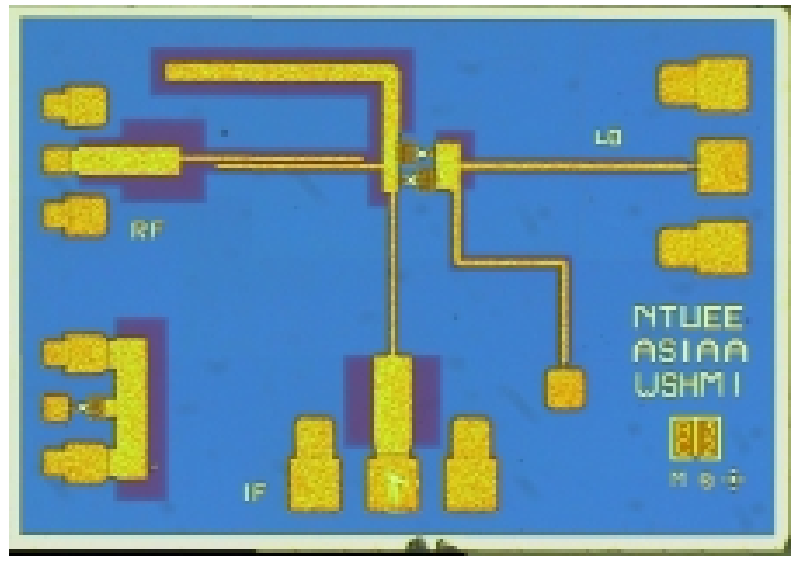

Figure 1:Photograph of WSHM1 SHP mixer MMIC chips.. The design has the RF port on the left side, LO port on the right side, and the IF port on the bottom side. 


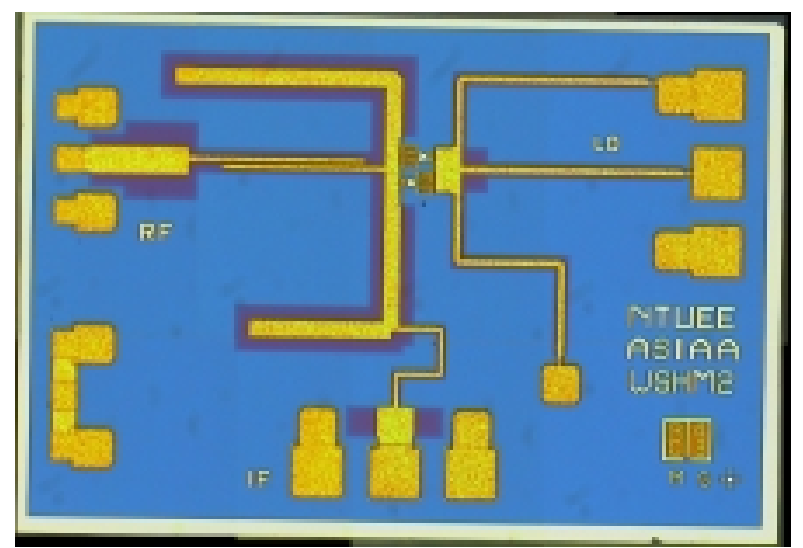

Figure 2: Photograph of WSHM2 SHP mixer MMIC chips. Individual port orientations are the same as WSHM1.

\section{CIRCUIT PERFORMANCE}

\section{Simulation}

The SHP mixers are simulated using HP/EEsof harmonic balance analysis. The critical matching circuit structures, such as the RF parallel coupled line band-pass filter, four- and five-port junctions connected to the diode pars, and microstrip tees junctions were analyzed numerically using Sonnet EM-software to ensure reasonable accuracy.

The simulated conversion loss of the WSHM1 mixer over $85-102 \mathrm{GHz}$ RF frequencies is about 12.5-17.5 dB with 7.0-dBm 42-GHz LO power. The simulation results for the WSHM2 mixer design show that the conversion loss is about 9 to $11 \mathrm{~dB}$ across $85-105 \mathrm{GHz}$, as a 7.0-dBm 42-GHz LO signal is pumped.

Simulation of the return loss gives about $5-11 \mathrm{~dB}$ at RF input port over $85-105 \mathrm{GHz}, 15-18 \mathrm{~dB}$ at LO port with $42-\mathrm{GHz}$ frequency and better than $7 \mathrm{~dB}$ at IF port over 1-21 GHz, for both WSHM1 and WSHM2. Simulation data also shows that the triple-frequency LO to RF (3xLO-RF) isolation is $40 \mathrm{~dB}$ over $36-50 \mathrm{GHz}$, and with 7-8 $\mathrm{dBm}$ LO power the LO-IF and LO-RF isolations are about 20 to $25 \mathrm{~dB}$ and $36 \mathrm{~dB}$, respectively.

\section{Conversion Loss Measurement}

The SHP conversion loss is measured with $-17 \mathrm{dBm}$ RF input power provided by a W85104A test set of an Agilent $85106 \mathrm{D}$ vector network analyzer, which operated in $\mathrm{CW}$ single-frequency mode as an extended W-band synthesizer over 68-118 GHz. A Q-band power source composed by a WR-22 mechanically-tunable Gunn oscillator and waveguide attenuators is used to provide LO signal at $42 \mathrm{GHz}$, one of the calibrated variable attenuator is used to adjust the LO power to appropriate level. The IF output spectrum was examined by a spectrum analyzer to ensure the unwanted spurious output 10-15 dB lower than the IF signal, then measured the IF output power by a power sensor, through a calibrated 0.01-20 GHz low-noise amplifier. Beyond $20 \mathrm{GHz}$, the unwanted spurious output level become significant so the IF power is measured directly by spectrum analyzer. The conversion loss measured results, as shown on Figure 3 and Figure 4 below, are 9.8-12.0 $\mathrm{dB}$ for WSHM2 under a 7.0-dBm LO power and 10.6-14.3 dB for WSHM1 under 8.5-dBm LO power.

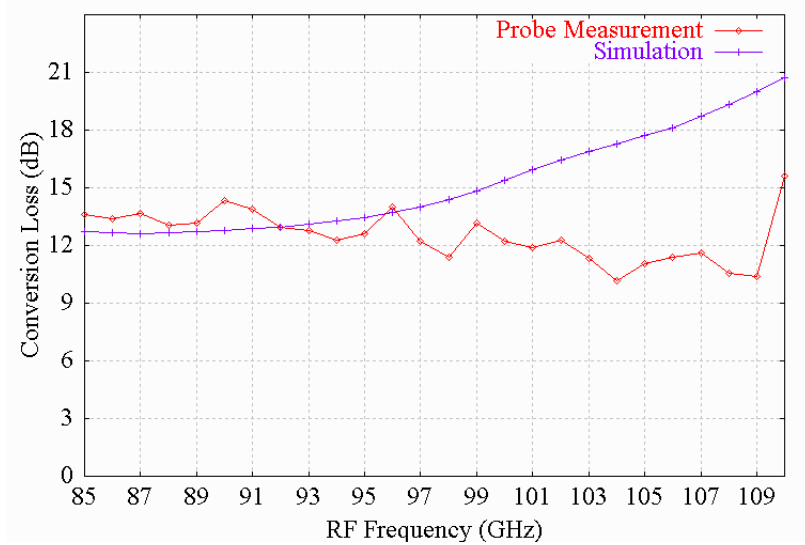

(a)

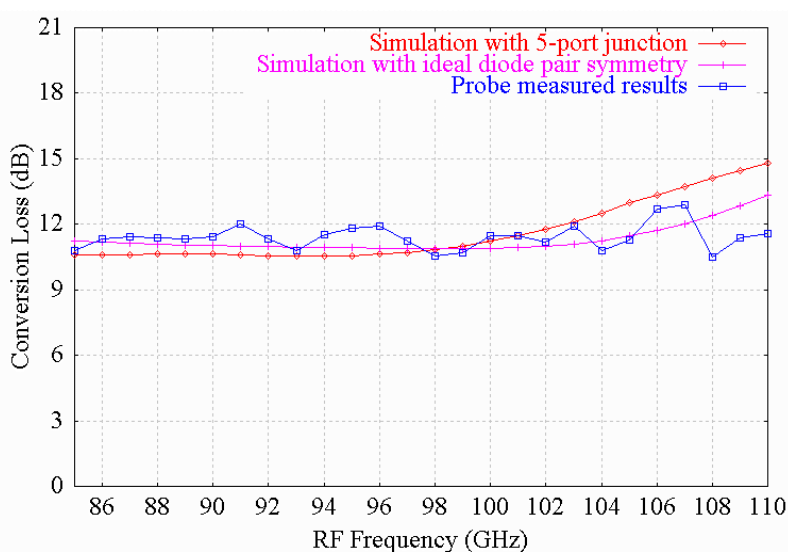

(b)

Figure 3: Conversion loss of WSHM1 (a) and WSHM2 (b) SHP diode mixers. RF frequency range is 85 to 110 $\mathrm{GHz} .42 \mathrm{GHz}$ LO pump level are with $8.5 \mathrm{dBm}$ and $7.1 \mathrm{dBm}$, respectively.

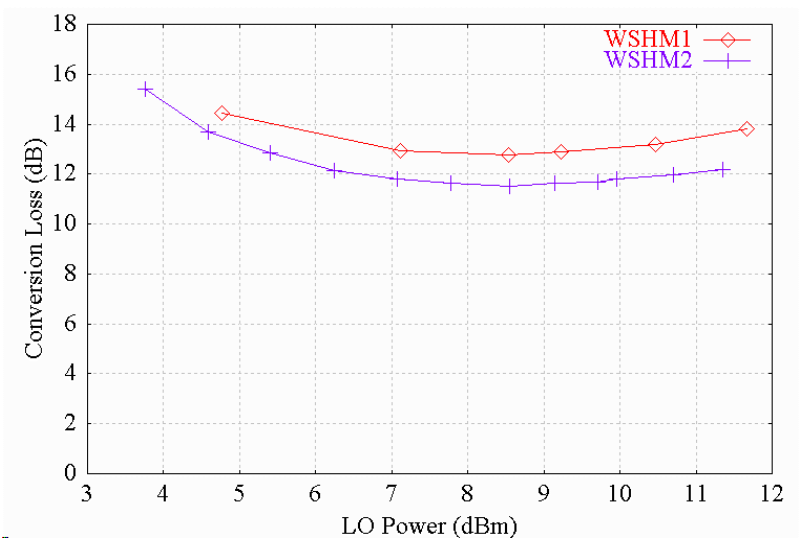

Figure 4: Relations between the LO power level and the measured conversion loss. RF frequency is set to $93 \mathrm{GHz}$ and the LO frequency is fixed at $42 \mathrm{GHz}$. 


\section{Return Loss and Isolation Measurement}

The return losses of the RF, LO and IF ports are measured by a vector network analyzer with one-port calibration. The results are shown on Figure 5, Figure 6, and Figure 7.

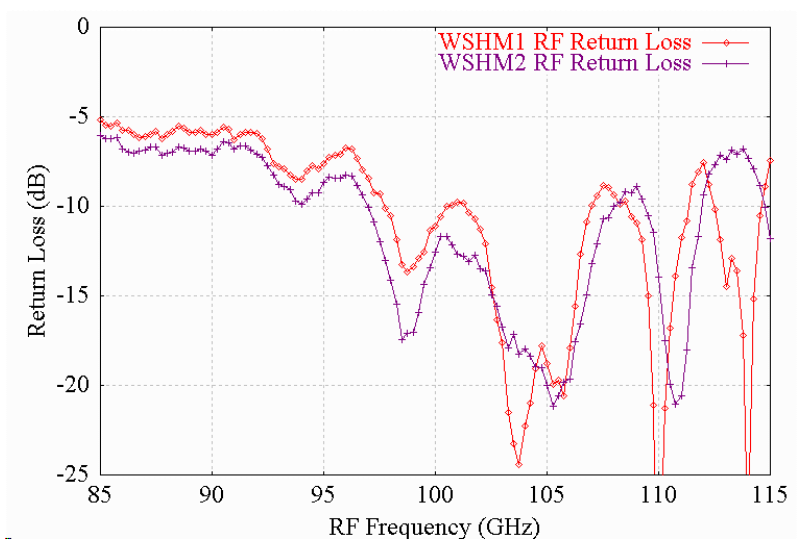

Figure 5: RF return loss measurement results.

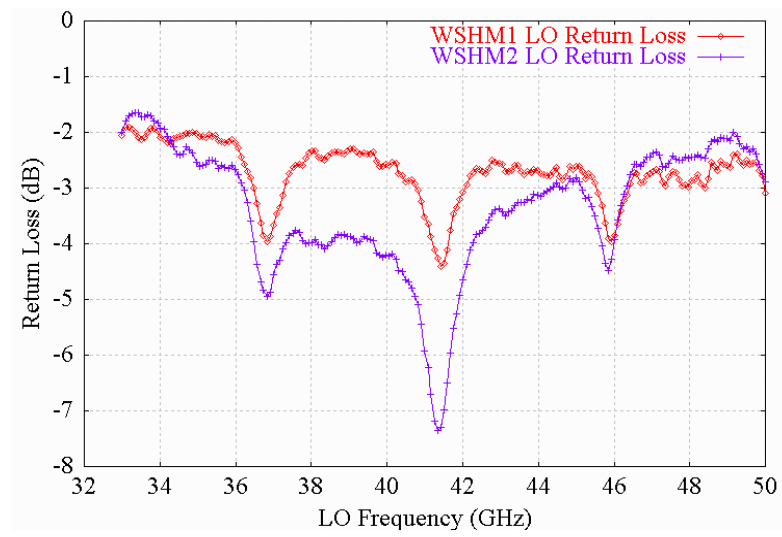

Figure 6: LO return loss measurement results. Please note that due to the low output power capability of VNA, the power pumped to the $L O$ port is $-23.8 \mathrm{dBm}$.

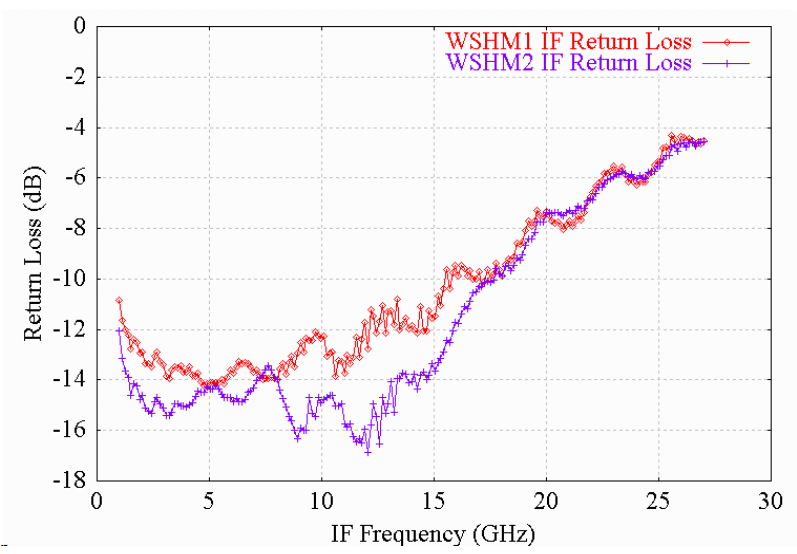

Figure 7: IF return loss measurement results. $R F$ is terminated with a WR-10 terminator through a GSG probe and the $L O$ power level is $7.5 \mathrm{dBm}$.

LO-IF isolation is measured by both power meter at 42 $\mathrm{GHz}$ with sufficient LO input power and VNA through a medium power amplifier from 33 to $50 \mathrm{GHz}$, with $\mathrm{RF}$ port with WR-10 waveguide terminator through GSG probe. LO-RF isolation is measured at $33-50 \mathrm{GHz}$ by VNA. Figure 8 shows the VNA measured results of LO IF and LO-RF isolation. The $3 \times$ LO-RF isolation is measured by WR-10 power sensor connected to RF port through GSG probe and IF port terminated. The results at $42-\mathrm{GHz}$ LO frequency are $25 \mathrm{~dB}$ for WSHM1 and 27 $\mathrm{dB}$ for WSHM2, respectively.

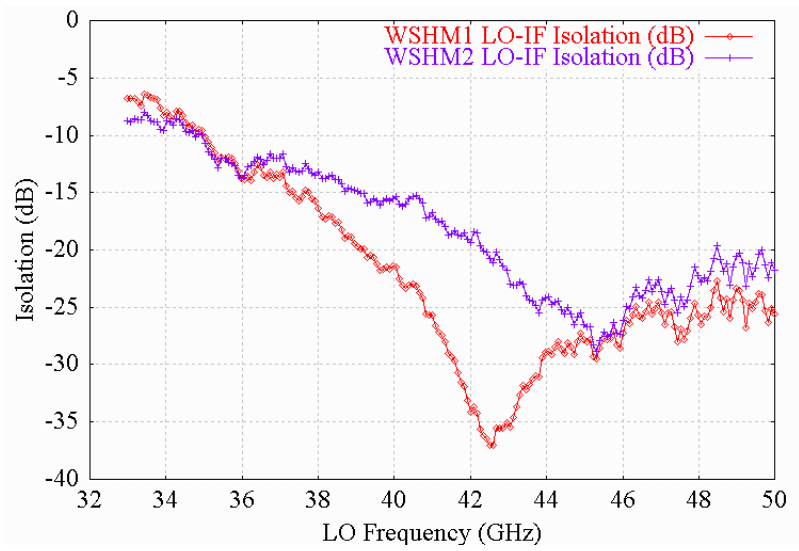

(a)

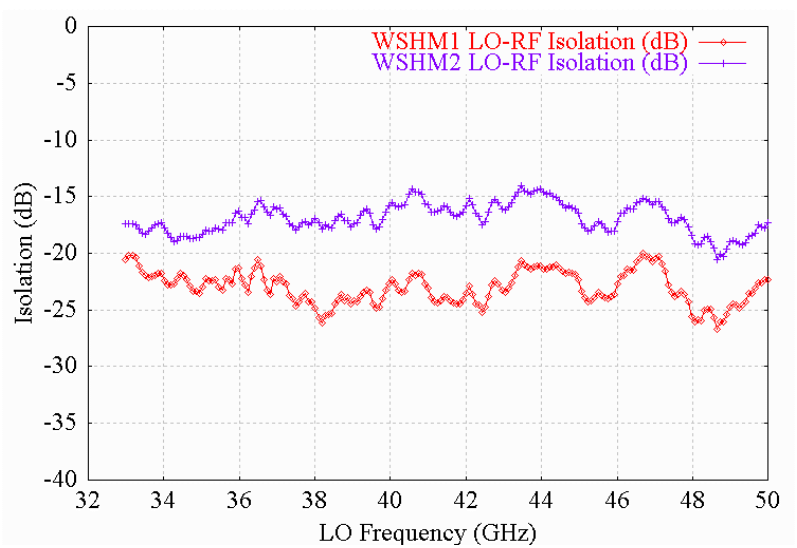

(b)

Figure 8: Measured transmission coefficients of (a) LO to IF port and (b) LO to RF port. Both were done by VNA, with $L O$ power level at about $1.4 \mathrm{dBm}$.

\section{Circuit Performance Summary}

The performance of these two SHP diode mixers, in comparison between simulation and measurement results, is listed in Table 1. From the results one can find that the comprehensive RF-IF diplexer with better isolation can improve the mixer conversion loss about $0.8 \mathrm{~dB}$.

\section{CONCLUSION}

The design and test of W-band broadband SHP MMIC diode mixers using 0.1- $\mu \mathrm{m}$ GaAs PHEMT process is presented. The measured down-conversion loss shows a good performance with IF frequency wider than $20 \mathrm{GHz}$.

\section{ACKNOWLEDGMENT}

This work is supported by Ministry of Education under 
Grant 89-N-FA-01-4-1 and 89E-FA06-2-4-6. The authors would like to thank Dr. Y. Z. Juang in Chip Implementation Center for the coordination effort on providing TRW GaAs MMIC foundry service.

\section{References}

[1] T. G. Phillips and J. Keene, "Submillimeter astronomy," Proceedings of the IEEE, vol. 80, No. 11, pp. 1662-1678, Nov. 1992.

[2] P. Kangaslahti, T. Gaier, D. Dawson, J. Tuovinen, T. Karttaavi, M. Lahdes, N. J. Hughes, T. L. Cong, P. Jukkala, P. Sjoman, and S. Weinreb, "Low noise amplifiers in InP technology for pseudo correlating millimeter wave radiometer," 2001 IEEE MTT-S Int. Microwave Symp. Dig., June 2001.

[3] N. L. Erickson, R. L. Grosslein, R.B. Erickson, S. Weinreb, "A cryogenic focal plan array for $85-115 \mathrm{GHz}$ using MMIC preamplifiers," IEEE Trans. Microwave Theory Tech., Vol. MTT-47, No. 12, pp. 2212-2219, Dec. 1999.

[4] M. W. Pospieszalski, E. J. Wollack, N. Bailey, D. Thacker, J. Webber, L. D. Nguyen, M. Le, and M. Lui, "Design and performance of wideband, low-noise, millimeter-wave amplifiers for microwave anisotropy probe radiometers," 2000 IEEE MTT-S Int. Microwave Symp. Dig., June 2000.
[5] M. T. Chen, Y. -J. Hwang, M. S. Ho, H. -M. Jiang, T. -H. Chu, S. -C. Lu, and M. W. Sinclair, "Full-polarization $W$-band receiver for CMB detection," Proceedings of SPIE Conference on Millimeter and Submillimeter Detector for Astronomy, paper \# 4855-38, August, 2002.

[6] Y.-L. Kok, H. Wang, M. Barsky, R. Lai, M. Sholley, and. B. Allen, "A 180-GHz monolithic sub-harmonic InP-based HEMT diode mixer, " IEEE Microwave and Guided Wave Letters, vol. 9, No. 12, pp. 529-531, Dec. 1999.

[7] Y.-L. Kok, P.-P. Huang, H. Wang, B.R. Allen, M. Sholley, T. Gaier and I. Mehdi, " 120 and $60 \mathrm{GHz}$ monolithic InP-based HEMT diode sub-harmonic mixer," 1998 IEEE MTT-S Int. Microwave Symp. Dig., vol. 1, pp. 43-46, June 1998.

[8] S. Raman, F. Rucky and G. M. Rebeiz, "A high-performance W-band uniplanar subharmonic mixer," IEEE Trans. Microwave Theory Tech., Vol. MTT-45, No. 6, pp. 955-962, June 1997.

[9] A. Madjar, "A novel general approach for the optimum design of microwave and millimeter wave subharmonic mixers," IEEE Trans. Microwave Theory Tech., Vol. MTT-44, No. 11, pp. 1997-1999, Nov. 1996.

[10] Y. -J. Hwang, C. -H. Lien, Huei Wang, R. G. Gough, M. W. Sinclair, H. Kanuniuk and T. -H. Chu, "A 78-114GHz monothilic subharmonically pumped GaAs-based HEMT diode mixer," IEEE Microwave and Wireless Device Letter, Vol.12, No. 6, pp. 209-211, June 2002.

Table 1

Summary of SHP diode mixers performance

\begin{tabular}{l|c|c|c|c}
\hline & \multicolumn{2}{|c|}{ WSHM1 } & \multicolumn{2}{c}{ WSHM2 } \\
\hline & Simulation & Measurement & Simulation & Measurement \\
\hline RF Frequency Range & $85-102 \mathrm{GHz}$ & $85-105 \mathrm{GHz}$ & $85-106 \mathrm{GHz}$ & $85-110 \mathrm{GHz}$ \\
\hline LO Frequency & $42 \mathrm{GHz}$ & $42 \mathrm{GHz}$ & $42 \mathrm{GHz}$ & $42 \mathrm{GHz}$ \\
\hline LO Power & $7 \mathrm{dBm}$ & $8.5 \mathrm{dBm}$ & $7 \mathrm{dBm}$ & $7.1 \mathrm{dBm}$ \\
\hline IF Frequency & $16 \mathrm{GHz}$ & $25 \mathrm{GHz}$ & $22 \mathrm{GHz}$ & $26 \mathrm{GHz}$ \\
\hline Conversion Loss & $12.5-17.5 \mathrm{~dB}$ & $10.2-14.3 \mathrm{~dB}$ & $9-11 \mathrm{~dB}$ & $10.5-13.0 \mathrm{~dB}$ \\
\hline RF Matching & $<-5.0 \mathrm{~dB}$ & $<-4.8 \mathrm{~dB}$ & $<-10.0 \mathrm{~dB}$ & $<-6.0 \mathrm{~dB}$ \\
\hline LO Matching frequency & $42 \mathrm{GHz}$ & $41.5 \mathrm{GHz}$ & $42 \mathrm{GHz}$ & $41.33 \mathrm{GHz}$ \\
\hline IF Matching $(\mathrm{f}<21 \mathrm{GHz})$ & $<-7 \mathrm{~dB}$ & $<-7 \mathrm{~dB}$ & $<-8 \mathrm{~dB}$ & $<-7 \mathrm{~dB}$ \\
\hline LO-IF Isolation $\left(\mathrm{P}_{\mathrm{LO}}=7.5 \mathrm{dBm}\right)$ & $>20 \mathrm{~dB}$ & $21.03 \mathrm{~dB}$ & $>25 \mathrm{~dB}$ & $26.05 \mathrm{~dB}$ \\
\hline LO-RF Isolation $\left(\mathrm{P}_{\mathrm{LO}}=1.4 \mathrm{dBm}\right)$ & $36 \mathrm{~dB}$ & $20 \mathrm{~dB}$ & $36 \mathrm{~dB}$ & $15 \mathrm{~dB}$ \\
\hline $3 \times L O-R F$ Isolation & $40.5 \mathrm{~dB}$ & $25.2 \mathrm{~dB}$ & $41.0 \mathrm{~dB}$ & $27.4 \mathrm{~dB}$ \\
\hline
\end{tabular}

